\title{
Nouveaux instruments pour la connaissance des champs de vitesses et de concentrations.
}

\author{
F. Larrarte $^{(\mathrm{a})}$, L-M Cottineau ${ }^{(\mathrm{b})}$ \\ (a)chercheur, Division eau; (b) chercheur, \\ Service Métrologie et Instrumentation Laboratoire Central des Ponts et Chaussées \\ route de Bouaye, BP 4129, \\ 44341 Bouguenais Cedex, \\ France frederique.larrarte@lcpc.fr
}

\section{Résumé}

Les prescriptions réglementaires et les impératifs techniques nécessitent une meilleure connaissance du fonctionnement réel des réseaux d'assainissement. Cette connaissance passe par celle de l'hydraulique des écoulements et par celle des polluants, notamment sous forme solide, transportés par ces derniers. Elle dépend de la capacité d'un point de mesure à donner des résultats représentatifs, et nécessite la définition de critères facilitant le choix de sites de mesure potentiels. Cet article présente les échantillonneurs des champs de vitesses et de concentrations conçus, développés et utilisés. Il présente aussi les champs de vitesses et de concentrations qui ont été obtenus à partir des mesures effectuées.

\begin{abstract}
Measuring flow-rate and pollutant discharges through sewer networks is required for legal, technical and management reasons. In order to achieve such measurements, a precise knowledge of the hydrodynamic conditions of each candidate measurement location proves critical. The LCPC has thereby become involved in a program on velocity and suspended matter concentration fields. This paper presents the devices developed to obtain 2D sampling of the velocities and pollutant concentrations.
\end{abstract}

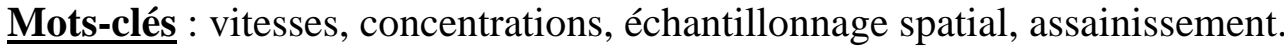

\section{Introduction}

Les écoulements en réseaux d'assainissement ne sont peut être pas les plus étudiés, pourtant une connaissance précise des flux polluants est importante pour concevoir et gérer ces ouvrages. D‘ailleurs, les Arrêtés du 24 décembre 1994 pris en application de la Loi sur l'Eau prescrivent que les systèmes de collecte doivent être muni de points de mesures qui doivent notamment permettre d'évaluer les rejets de polluants au milieu naturel. Ces textes préconisent d'une part une mesure des débits liquides transitant dans les collecteurs et d'autre part une estimation des polluants transportés par l'eau. Les collecteurs étant considérés comme monodimensionnels, on cherche à obtenir une valeur moyenne de la vitesse et de la concentration dans une section, et on les assimile aux résultats fournis par un échantillonnage limité à un seul point (préleveur), ou à un volume plus ou moins bien défini (vélocimètre Doppler). Il en résulte des mesures assez imprécises. Or des travaux relatés dans la littérature (Wohrle et Brombach, 1991) invitent à étudier la répartition des vitesses et des polluants à travers toute la section. 
Un programme de recherches combine actuellement des études expérimentales et numériques afin d'appréhender la répartition des vitesses et des concentrations dans une section, située dans un environnement donné et soumise à des sollicitations hydrauliques connues, pour vérifier la représentativité des capteurs qui peuvent y être implantés (vélocimètres, préleveurs). Le but est de proposer un protocole permettant de qualifier des sections de mesure, et, connaissant le domaine échantillonné par un capteur donné, de définir le traitement à appliquer aux mesures brutes pour obtenir les meilleures estimations possibles des mesurandes (Larrarte et al., 2004) ${ }^{2}$.

$\mathrm{La}$ principale conséquence de ces objectifs est que l'on a besoin de vues bi ou tridimensionnelles des champs de vitesses et de polluants. Cet article s'intéresse aux travaux expérimentaux dans une section droite de collecteur. Des extrapolations tridimensionnelles ont été faites avec un code de calcul (Jaumouillié, 2003)

\section{Inventaire des dispositifs existants}

Pour les vitesses, le dispositif présenté par (Hugues et al., 1996) ${ }^{4}$ réalise un échantillonnage spatial du champ de vitesses en utilisant un réseau de capteurs Doppler à poste fixe. Nous n'avons pas retenu ce système car le nombre de points de mesure est encore limité et leurs positions ne peuvent être aisément réglées. Des capteurs pulsés à effet Doppler, issus de techniques développées en océanographie et en hydraulique fluviale, ont récemment été développés pour des applications en collecteur mais ils ont une faible résolution spatiale et ce sont plutôt des échantillonneurs 1D.

Du point de vue des concentrations, ce sont les polluants associés aux particules en suspension qui posent un problème d'échantillonnage, la phase dissoute étant homogène. (Ashley et al., 1994) ${ }^{5}$, (Ahyerre, 1999) ${ }^{6}$ ont effectué des prélèvements en plusieurs points mais ils s'intéressaient aux couches très concentrées en matières solides qui forment le dépôt solide présent au fond de certains collecteurs. (Wohrle et Brombach, 1991) ${ }^{1}$ et (Verbanck, 1993) ${ }^{7}$ ont effectué des échantillonnages spatiaux des solides transportés en suspension mais uniquement par temps sec. Or non seulement les débits sont plus importants par temps de pluie mais c'est également lors des évènements pluvieux que le réseau risque de déverser au milieu naturel. Les dispositifs issus de l'hydraulique fluviale tels que celui de (Dijkman et al., 1982) ${ }^{8}$ ou les bouteilles Ottman ont une taille incompatible avec leur maniement depuis les regards.

\section{Développement d'échantillonneurs 2D}

La principale contrainte vient des règles de sécurité qui interdisent l’accés aux collecteurs en temps de pluie. Les mesures doivent donc pouvoir être faites alors que seul le regard de visite (dont le diamètre est typiquement de $1 \mathrm{~m}$ ) est accessible. Ceci implique que chaque élément du montage soit aussi maniable que possible puisqu'une partie de celui-ci doit être apportée sur place à chaque expérimentation. Les équipements qui restent à poste dans le collecteur doivent être situés hors d'eau la plupart du temps, et ils doivent résister à la corrosion.

La deuxième contrainte vient du contexte hydraulique. La figure 1 montre un exemple de données enregistrées sur site. On peut noter que par temps sec les hauteurs et vitesse de l'eau suivent un cycle quotidien alors que dés qu'un événement pluvieux survient, les débits et donc les hauteurs et les vitesses des effluent varient considérablement. 
Il faut donc que les parties du montage restant dans l'émissaire puissent supporter des immersions occasionnelles et des vitesses supérieures à un, voire plusieurs, mètre par seconde, que les capteurs utilisés aient une gamme étendue et que l'échantillonnage spatial permette de balayer une surface mouillée et plusieurs mètre carrés. En outre, l'idéal serait que toutes les mesures puissent être faites simultanément. A défaut il convient de les réaliser dans un laps de temps minimum.

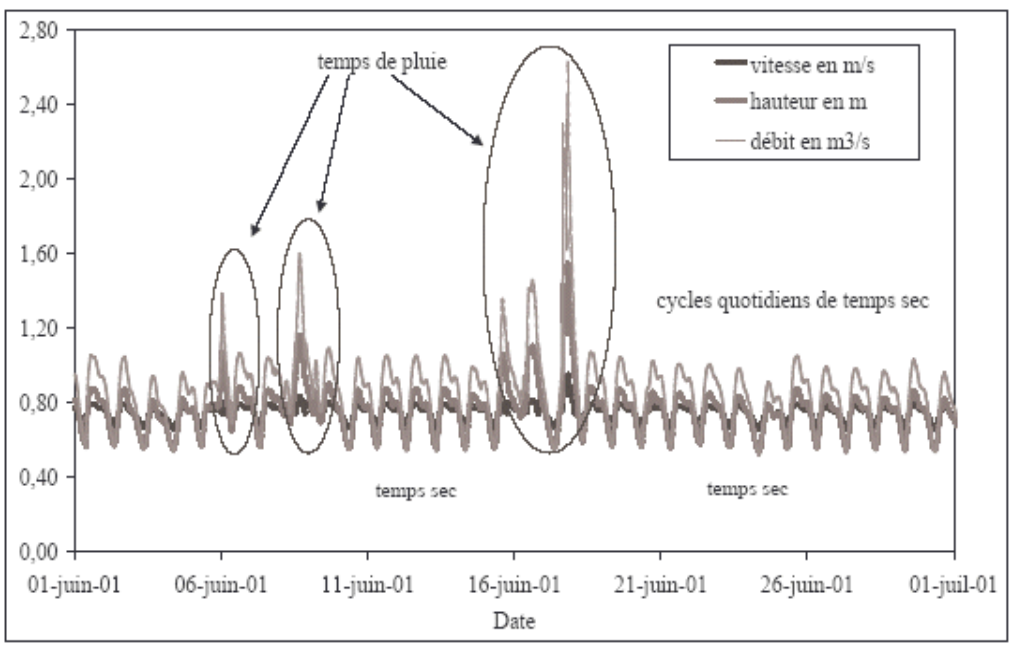

figure 1: Gammes de hauteurs, vitesses, débits rencontrés sur le site

Le choix a été fait de scinder le problème en deux : le champ de vitesses est exploré avec un dispositif baptisé Cerbère, le champ de concentrations est échantillonné avec un autre système baptisé Orphée.

\subsection{Echantillonnage des vitesses}

Afin d'obtenir une carte de vitesses dans une section droite normale à l'écoulement, cette dernière est discrétisée en points de mesure. Pour cela Cerbère (figure 2) permet de mesurer simultanément les vitesses en trois points situés sur une verticale avec 3 vélocimètres ultrasonores à effet Doppler. Ces capteurs sont fixés tous les $20 \mathrm{~cm}$ sur un support vertical. Le déplacement des capteurs est contrôlé depuis l'extérieur du collecteur à l'aide d'un chariot (déplacement horizontal) et d'un double vérin (déplacement vertical). Il est ainsi possible d'obtenir un profil vertical de vitesses sur toute la hauteur d'eau puis, en réitérant cette démarche en déplaçant le chariot, l'échantillonnage bidimensionnel du champ de vitesses peut être obtenu. Il est possible de discrétiser toute la section droite depuis le fond jusqu'à 1,50 m de haut, ce qui correspond à 90\% des hauteurs d'eau observées sur une année sur le site d'essai retenu. En chaque point la mesure est effectuée deux fois, et chaque mesure est la moyenne des vitesses instantanées enregistrées pendant 10 secondes. Avec cette méthode il faut de 45 à 80 minutes pour effectuer toutes les mesures nécessaires à la constitution d'une carte. 


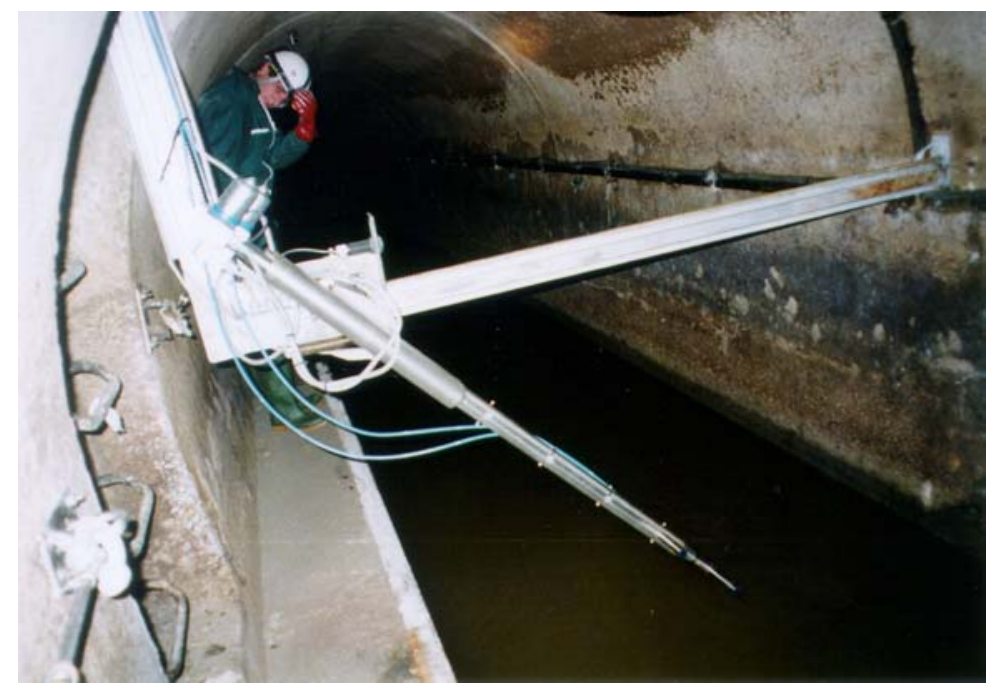

Figure 2. Cerbère : l'échantillonneur $2 D$ du champ des vitesses quand il rentre dans le collecteur

\subsection{Echantillonnage des concentrations}

Il est évident que plus il y a de points de prélèvements et plus les résultats sont représentatifs du champs des solides transportés en suspension dans l'écoulement. Cependant, tous les échantillons doivent pouvoir être analysés durant la même demi-journée et aussitôt que possible afin d'éviter toute modification chimique. En tenant compte de tout cela le préleveur bidimensionnel Orphée a été conçu et fabriqué. Il permet jusqu‘à 24 prélèvements simultanés dans une section droite de collecteur. Les échantillons sont analysés au laboratoire afin d'obtenir les concentrations. Orphée (figure 3) est composé de 4 faisceaux verticaux de 6 tubes en inox. Chaque tube est connecté à un tuyau en plastique qui remonte à la surface. Chaque faisceau pivote autour d'un axe fixé à la largeur maximale du collecteur (en l'occurrence à 1,76 m du radier). Comme pour les vitesses, il est possible de discrétiser toute la section droite depuis le fond jusqu'à $1,50 \mathrm{~m}$ de haut. Les prélèvements sont effectués au moyen de bouteilles de 2,5 1 dans lesquelles le vide a préalablement été fait. S‘il faut moins de $5 \mathrm{mn}$ pour effectuer tous les prélèvements à partir du moment où les faisceaux sont reliés aux bouteilles et abaissés dans l'écoulement, il faut par contre environ une minute de préparation par point de prélèvement (connections des tuyaux et vide dans la bouteille) avant de pouvoir réitérer l'opération. 


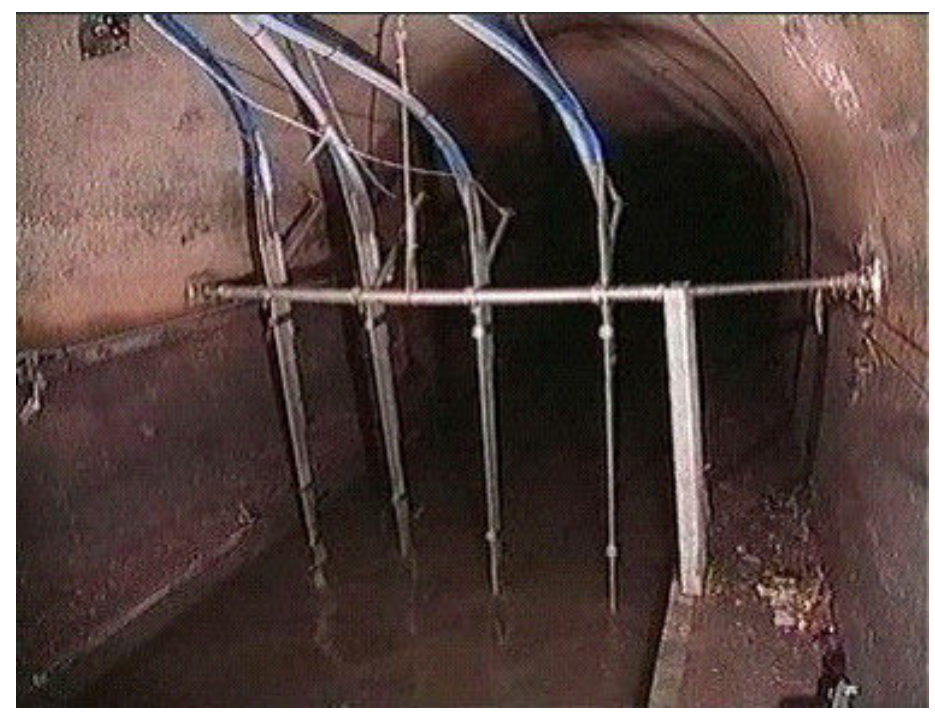

Figure 3. Orphée : échantillonneur 2D du champ de concentrations, en cours d'abaissement

\section{$\underline{\text { 4.Résultats }}$}

Les résultats obtenus avec les outils expérimentaux décrits ci avant ont été comparés avec ceux obtenus avec des appareils de mesure plus traditionnels afin de tester leur capacité à déterminer les valeurs moyennes dans une section. Il convient de garder à l'esprit que, bien que ces campagnes de mesures aient été effectuées avec soin, il s'agit de travaux effectués dans un collecteur réel du réseau d'assainissement de la Communauté Urbaine de Nantes. Il est notamment impossible d‘éviter que des macro déchets viennent encrasser les capteurs.

\subsection{Cartes de vitesses}

Deux mesures de vitesses sont effectuées successivement en chaque point. Si l'écart entre ces deux mesures est supérieur à $0,05 \mathrm{~m} / \mathrm{s}$ les capteurs sont nettoyés. Il arrive cependant parfois que de mauvaises mesures soient effectuées et dans certains cas aucune mesure n'a été possible, car les encrassements étaient trop fréquents. La figure 4 présente deux exemples de cartes de vitesses. Afin de tenir compte des variations de débits durant les mesures, les vitesses sont exprimées comme le ratio $\mathrm{Vi} / \mathrm{Vms}$ de la valeur Vi mesurée en un point à un instant donné par la vitesse de Manning Strickler calculée en fonction de la hauteur d'eau dans le collecteur. On peut noter l'existence de gradient de vitesses notamment près des parois et la présence de la vitesse maximale au centre de l'écoulement en dessous de la surface libre. Le calcul de la vitesse moyenne par la méthode des aires suivante :

$v_{m}=\frac{1}{S m} \sum\left(v_{i} S_{i}\right)$

où Sm est la surface mouillée de la section verticale et Vi la vitesse locale sur l'élément de surface $\mathrm{Si}$, a permis de montrer que le ratio $\mathrm{Vm} / \mathrm{Vms}$ augmente légèrement avec la hauteur d'eau et que la répétabilité est bonne (Jaumouillié et al., 2002) ${ }^{9}$. Sur ce site particulier, la formule de Manning Strickler calculée avec un coefficient de K=70 donne une bonne estimation de la vitesse moyenne. 


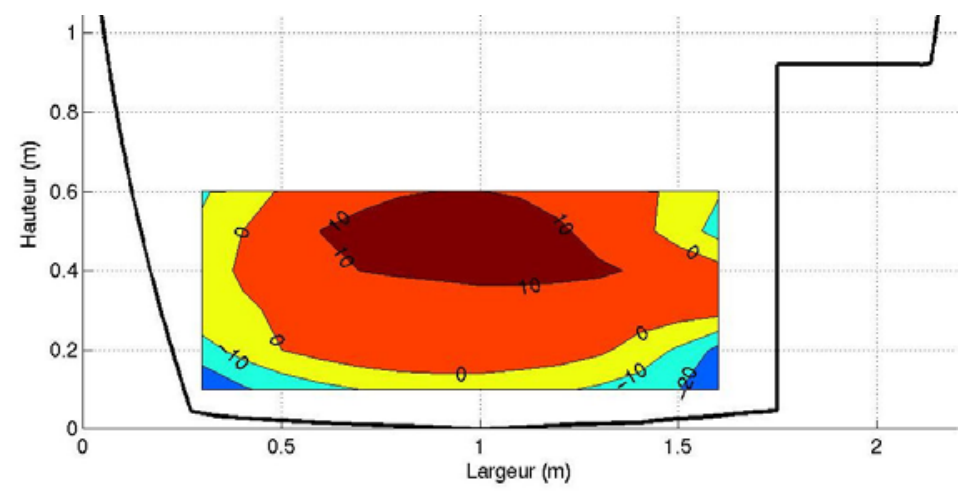

a) temps sec

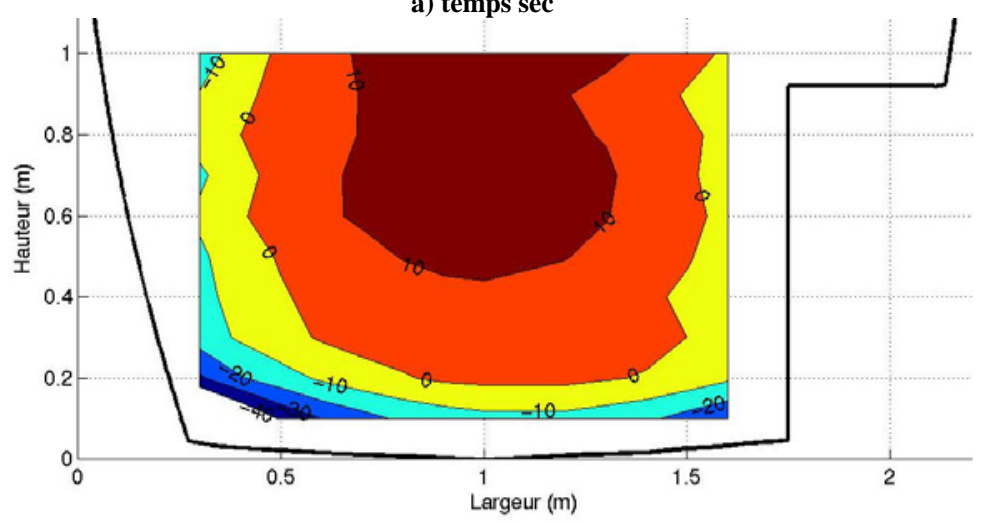

b) temps de pluie

Figure 4. Exemple d'iso cartes de vitesses Vi / Vms sur le site de Cordon Bleu.

\subsection{Cartes de concentrations}

L'échantillonneur 2D Orphée a permis de constituer un jeu de cartes de concentrations correspondant à des conditions météorologiques variées qui vont du temps très sec d'été au cas des fortes pluies orageuses. Quelques soient les conditions hydrauliques, aucune sédimentation n'a été observée sur ce site expérimental et il $n^{\prime} y$ a pratiquement pas de gradients de concentrations. En lissant les profils de vitesses par une loi logarithmique le cisaillement au fond a été évalué à $1 \mathrm{~N} / \mathrm{m} 2$. Pour les hauteurs d'eau inférieures à 0,95 m, les concentrations moyennes sont comprises entre 250 et $400 \mathrm{mg} / \mathrm{l}$. Cette gamme est plus étendue durant les périodes pluvieuses et dépend de la durée de la période de temps sec qui a précédé. La figure 5 montre l'influence de celle-ci puisque pour une même hauteur d'eau les concentrations sur le même site expérimental varient du simple au double. A gauche, la pluviométrie des 7 jours précédents était de $13 \mathrm{~mm}$ alors qu'elle était de $31 \mathrm{~mm}$ pour les 7 jours précédent le 13 décembre (figure de droite). De plus, alors que les débits transités sur ce site en décembre 2000 sont en moyenne de 76000 m3/j, les débits du 10 et du 12 décembre étaient supérieurs à 90000 m3/j. On observe donc une certaine dilution des effluents. 

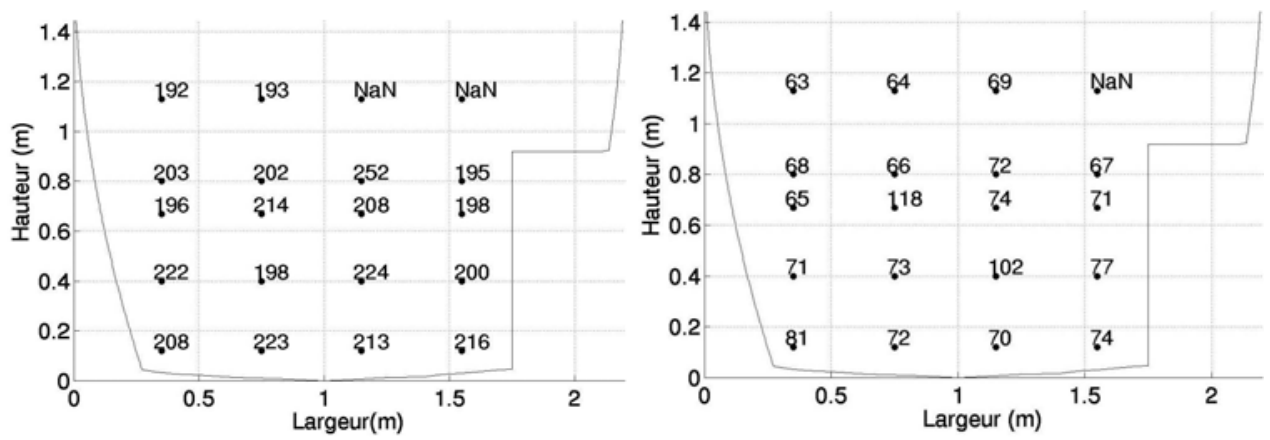

Figure 5 : Influence des antécédents de temps sec sur les cartes de concentrations.

\section{Conclusion}

Malgré quelques problèmes de fiabilité dus à l'atmosphère corrosive des réseaux, les échantillonneurs 2D Cerbère et Orphée nous ont permis de constituer des collections de cartes de vitesses et de concentrations dans une section droite d'un écoulement canalisé, celui d'un réseau d'assainissement. Ces cartes servent désormais à valider les résultats numériques obtenus dans le cadre du protocole visant à utiliser la mécanique des fluides numériques pour aider à l'instrumentation des réseaux. Par ailleurs, nous développons un dispositif permettant de mesurer simultanément et aux mêmes points les deux données.

\section{Remerciements}

Les auteurs remercient le GIP GEMCEA (Groupement pour l'Evaluation de la Mesure en Continu dans les Eaux et en Assainissement) pour le soutien apporté ainsi que les personnels du LCPC et de la Direction de l'Assainissement de la Communauté Urbaine de Nantes pour leur aide lors ces travaux.

\section{Références}

1 Wohrle C. and Brombach H., (1991), Probenahme im abwasserkanal, Wasserwirtschaft, Vol. 81, pp.60-65.

2 Larrarte F., Jaumouillié P., Joannis C., (2004), Computational Fluid Dynamics: an aid for designing the instrumentation of sewer sections. Novatech 2004, Tome1, pp.729-736.

3 Jaumouillié P., (2003), Hétérogénéités des vitesses et des concentrations dans les collecteurs d'assainissement : application à la mesure des flux polluants, Thèse-Université de Bordeaux 1, 233 pages,.

4 Hughes A.W., Longair I.M., Ashley R.M. and Kirby K., (1996), Using an array of ultrasonic velocity transducers to improve the accuracy of large sewer mean velocity measurements, Water Sci. and Tech., Vol. 33, No. 1., pp. 1-12.

5 Ashley R.M., Arthur S., Coghlan B.P. and McGregor I., (1994), Fluid sediment in combined sewers, Water Sci. and Tech., Vol. 29, No. 1-2, pp. 113-123.

6 Ahyerre M., (1999), Bilans et mécanismes de migration de la pollution organique en réseau d'assainissement unitaire, thèse -Université de Paris 6, 250 p. 
7 Verbank M.A. , (1993), Identification of the depth-dependent transportation of particulate solids in dry weather sewage flows, 6th Int. Conf. On Urban Storm Drainage, Niagara Falls, pp. 742-747.

8 Dijkman J.P., Milisic V., (1982), Investigations on suspended sediment samplers based on measurements in the Danube River., Delft Hydraulics Laboratory and Jaroslav Cerni Institute, S240, February, 39 pages + annexes.

9 Jaumouillié P., Larrarte F., Milisic V., (2002), New devices for 2D sampling of velocities and pollutant concentrations in sewers, 3rd Int. Conf. on sewer process and networks., pp. 171178. 